\section{Kidney Blood Pressure Research}

\title{
TMEM106a is a Novel Tumor Suppressor in Human Renal Cancer
}

\author{
Chenglong Wu Juan $\mathrm{Xu}^{\mathrm{b}}$ Han Wang ${ }^{\mathrm{a}}$ Jinghua Zhang ${ }^{\mathrm{b}}$ Jianhua Zhong \\ Xiaowen Zou ${ }^{b}$ Yan Chen ${ }^{b}$ Gang Yang ${ }^{d}$ Yuantang Zhong ${ }^{d}$ Daihuan Lai ${ }^{b}$ \\ Xuyi Li ${ }^{b}$ Aifa Tang ${ }^{b}$
}

aShenzhen Second People's Hospital, Guangzhou Medical University, Shenzhen, 'Department of Urinary Surgery, Shenzhen Second People's Hospital, the First Affiliated Hospital of Shenzhen University, Shenzhen, 'Clinical Medicine College of Anhui Medical University, Hefei, dGuangzhou medical university, Guangzhou, China

\section{Key Words}

TMEM106a • Renal cancer $•$ Apoptosis $•$ Cell migration $•$ Proliferation

\begin{abstract}
Background/Aims: In recent years the diagnosis and management of renal cancer has changed greatly, although the mechanism is still elusive. TMEM106a is a conserved type II transmembrane protein which is a key factor to regulate macrophage activation. Its inactivation in gastric cancer is frequently observed to be associated with poor prognosis. The role of TMEM106a in renal cancer remained unclear. Methods: TMEM106a expression profiling was performed in a panel of renal cancer cell lines and primary renal tissue cells. Then TMEM106a was overexpressed by a viral system in a renal cancer cell line with low level of TMEM106a. This stable cell line was assessed in multiple cell growth and migration assays. The results from TMEM106a overexpressing cell line were then confirmed with primary renal cells by siRNA knockdown of TMEM106a. Results: TMEM106a expression level was reduced in renal cancer cells compared to normal primary renal cells. Restoration of TMEM106a expression in TMEM106a-low renal cancer cells resulted in attenuated proliferation, reduced cell migration and enhanced caspase 3 dependent apoptosis compared to control cells. TMEM106a knockdown in primary renal cells led to increased colony formation compared to the control cells with scrambled siRNA transfection. Conclusion: TMEM106a is a novel tumor suppressor in renal cancer.
\end{abstract}

\section{Introduction}

The kidney is an essential organ that maintains the homeostatic balance of fluids and solutes in the human body, and removes waste products from the blood. It also helps to regulate $\mathrm{Wu}, \mathrm{Xu}$, Wang are contributed equally.

\begin{tabular}{ll}
\hline Aifa Tang & Department of Urinary Surgery, Shenzhen Second People's Hospital, \\
& First Affiliated Hospital of Shenzhen University, Shenzhen 518037 (P.R. China) \\
& E-Mail tangaifa2004@163.com
\end{tabular}


blood pressure, and secretes several hormones [1]. Adult kidney cancers that arise in the renal parenchyma are mainly adenocarcinomas, also known as renal cell carcinomas (RCC), while those that arise from the collecting system are mainly transitional cell carcinomas. RCC is the ninth most common cancer worldwide and the incidence of RCC is increasing annually, accounting for approximately 3.8\% of adult malignancies and $90-95 \%$ of neoplasms arising from the kidney in 2010 [2,3]. Up to $30 \%$ of RCC patients present at advanced stages, and approximately $40 \%$ of patients experience recurrence during subsequent follow-up after curative surgical resection [4]. RCC is characterized by a lack of early warning signs, diverse and variable clinical manifestations, resistance to radiation and chemotherapy [5-7]. Better understanding of the underlying mechanism holds the key for more effective renal cancer treatment. A bulky body of research studies have revealed the complicated pathophysiology of renal cancer. The most striking RCC factor is the loss of von Hippel-Lindau (VHL) tumor suppressor which is inactivated in all hereditary RCC and approximately $50 \%$ of sporadic RCC [8-10]. VHL is a key regulator of hypoxia induced factor (HIF) and its dysregulation is frequently associated with aberrant activation of p53, VEGF, mTOR, NFKB, MAPK pathways which collectively drive the tumor growth, apoptosis evasion, angiogenesis and metastasis [11-13]. To facilitate the development of therapy, it is of great importance to identify and characterize novel molecular targets of RCC.

Here we reported the critical role of TMEM106a (Transmembrane Protein 106a) gene as a new factor in the pathogenesis of RCC. TMEM106a is a type II membrane protein and has two close members TMEM106b and TMEM106c. It is conserved in human, chimpanzee, monkey, dog, mouse and rat. In mice TMEM106a is expressed in multiple tissues including liver, spleen, lung, kidney, lymph node, thymus and intestine [14]. At cellular level TMEM106a protein is localized to the plasma membrane. It is involved in macrophage polarization through activation of MAPK and NFKB pathways [15]. TMEM106b is localized in the late endosome and lysosome compartments and is regulated by lysosomal activities $[16,17]$. TMEM106b is reported to be associated with cognitive impairment in amyotrophic lateral sclerosis and in the pathological manifestation of Alzheimer's disease [18, 19]. Human TMEM106c is a differentially expressed transcript in ankylosing spondylitis [20], and porcine TMEM106c is a positional and functional candidate for arthrogryposis multiplex congenita [21]. Interestingly, loss or reduction of TMEM106a expression is frequently observed in gastric cancer and associated with promoter region hypermethylation. Restoration of TMEM106a expression induced gastric cancer cell apoptosis and suppressed tumor growth in xenograft model [14]. All these results convincingly suggest that TMEM106a is a tumor suppressor in gastric cancer.

In this study we have examined the role of TMEM106a gene in renal cancer, since it is also expressed in normal kidney tissue and regulates cell proliferation and migration. Our results demonstrated that TMEM106a was down-regulated in many renal cancer cell lines. Over-expressing TMEM106a in renal cancer cells inhibited cell proliferation, trans-well migration and activated caspase-dependent apoptotic pathway. Knocking down TMEM106a in normal renal cells promoted their clonogenic formation capacity. In summary, our findings collectively suggest TMEM106A functions as tumor suppressor in renal cancer.

\section{Materials and Methods}

\section{Cell culture, transfections and treatments}

Renal Cell Adenocarcinoma cell lines 786-0,769-P, ACHN, A-498, Adenocarcinoma cell line A-794, Primary Renal Proximal Tubule Epithelial Cells, Primary Renal Cortical Epithelial Cells were from American Type Culture Collection, USA, and were cultured in RPMI-1640 (Hyclone, USA) supplemented with 10\% (v/v) FCS (Hyclone), penicillin/streptomycin $(100 \mathrm{U} / \mathrm{mL}), \mathrm{L}$-glutamine $(2 \mathrm{mM})$ and 2-ME $\left(5 \times 10^{-5} \mathrm{M}\right)$.

Primary Renal Proximal Tubule Epithelial Cells were transfected with 600 pmol of siRNA using Lipofectamine 2000 reagent according to the manufacturer's instructions (Invitrogen, USA). Briefly, 


\section{Kidney Blood Pressure Research}

$600 \mathrm{pmol}$ of siRNA and $30 \mu \mathrm{L}$ of Lipofectamine 2000 were diluted with Opti-MEM to a final volume of $3 \mathrm{~mL}$, and added to primary renal epithelial cells grown to $60 \%$ confluence in $100 \mathrm{~mm}$ diameter plates. $6 \mathrm{~h}$ later, cells were washed and then cultured in fresh medium for $24 \mathrm{~h}$. Cells were then washed and analyzed to determine the effectiveness and specificity of siRNA treatment against Tmem106a.

\section{Lentiviral Transduction}

Full-length cDNA of TMEM106a was cloned into lentiviral vector system from GeneScript, China. Lentiviral particles containing empty vector control or TMEM106a cDNA were prepared with packaging system from Invitrogen, Carlsbad, CA, USA following the instruction of manufacturer. Viral particles were filtered and concentrated from cell medium supernatant. After titration, viral transduction was performed in the presence of $6 \mu \mathrm{g} / \mathrm{ml}$ Polybrene for $24 \mathrm{~h}$. Then the cells were cultured in fresh medium for $24 \mathrm{~h}$ after removing the virus-containing supernatant.

\section{Cell viability assay}

Cells infected with Lenti-null or Lenti-TMEM106A were harvested and plated in 96-well plates at 2000 cells per well and incubated at $37^{\circ} \mathrm{C}$. The lentiviral vector backbone is Lenti-X expression system (EF1alpha) from Clontech. Cell viability was analyzed using the Cell Counting Kit-8.

Trans-well cell migration assay

Cells with different level of TMEM106a were seeded at $5 \times 10^{5} /$ well density to the upper chamber of trans-well (Corning, NY, USA). After $24 \mathrm{~h}$ incubation, cells migrated to the bottom plate were fixed and stained with Toluidine Blue $\mathrm{O}$ dye following the instructions of manufacturer.

\section{Colony formation assay}

Cells were transfected with TMEM106a siRNA or control siRNA using lipofectamine 2000 . After $48 \mathrm{~h}$, cells were seeded at $1 \times 10^{5}$ cells/well density and incubated at $37^{\circ} \mathrm{C}$ for 5 days. Colonies with $\geq 50$ cells were counted after fixation and crystal violet staining.

\section{RT-PCR assay}

Total cellular RNA samples from cell lines were extracted using the Trizol ${ }^{\mathrm{TM}}$ Reagent (Invitrogen, USA). RT-PCR was performed using the ThermoScript RT-PCR System (Invitrogen, USA). Primers used were listed as below:

TMEM106a:
GCCTCTGTCCTTTCTGGTTATT (F)
GCAGCTGGATACTGATAG (R)
GAPD: ACCCACTCCTCCACCTTTGA (F)
CTGTTGCTGTAGCCAAATTCGT (R)

\section{Western blotting}

Total protein from cells or mouse tissues was extracted using RIPA buffer (20 mM Tris-HCl pH 7.5, 150 $\mathrm{mM} \mathrm{NaCl}, 1 \mathrm{mM}$ EDTA, 1 mM EGTA, 1\% NP-40, 1\% sodium deoxycholate, $2.5 \mathrm{mM}$ sodium pyrophosphate, $1 \mathrm{mM}$ $\beta$-glycerophosphate, $1 \mathrm{mM} \mathrm{Na} 3 \mathrm{VO} 4,1 \mu \mathrm{g} / \mathrm{ml}$ leupeptin). Following solubilization, proteins were quantified using a BCA protein quantification kit (Thermo Scientific, Rockford, IL, USA). 40 micrograms of protein was loaded into each well and separated on a 10\% SDS Tris-Glycine gel, then transferred onto polyvinylidene difluoride (PVDF) membranes (Invitrogen, USA) at a constant current of $250 \mathrm{~mA}$ in transfer buffer (50 mM Tris, pH 8.0, 0.192 M glycine, 20\% (v/v) methanol), using a Bio-Rad Trans-Blot Cell (Bio-Rad, Hercules, CA, USA). The membranes were incubated for $1 \mathrm{~h}$ at room temperature in blocking buffer (TBS containing $5 \%$ non-fat milk), followed by an overnight incubation at $4{ }^{\circ} \mathrm{C}$ with appropriate primary antibodies in blocking buffer. After three washes with TBS containing 0.05\% Tween 20, strips were incubated for $1 \mathrm{~h}$ with corresponding HRP-conjugated secondary antibodies. Protein-antibody complexes were visualized using an ECL detection system as recommended by the manufacturer (Applygen Technologies, Beijing, China). Data were recorded using a Bio-Rad Gel Doc 2000 system. Anti-TMEM106a antibody was purchased from Abcam (ab140192), and Caspase3 (\#9662) and GAPDH (\#5174) antibodies were from Cell Signaling. 


\section{Kidney Blood Pressure Research}

\begin{tabular}{l} 
Kidney Blood Press Res 2017;42:853-864 \\
\begin{tabular}{l|l} 
DOI: 10.1159/000484495 & (c) 2017 The Author(s). Published by S. Karger AG, Basel \\
published online: October 31, 2017 & ww.karger.com/kbr
\end{tabular} \\
\hline
\end{tabular}

Wu et al.: Role of TMEM106a in Renal Cancer

\section{Flow cytometry}

To detect the apoptosis in the cells after overexpressing TMEM106a with lentivirus, cells were harvested and analysed with a FITC-Annexin V staining Detection kit (Biosea Biotechnology, Beijing, China) on a FACSCalibur flow cytometer according to the manufacturer's instructions.

\section{Statistical analysis}

All experiments described here have been repeated at least three times. Results are presented as mean \pm standard deviation (SD). Comparison of the data was performed using Student's $t$ test. Significance was defined as $p<0.05$. Statistical analysis was performed using SPSS software.

\section{Results}

TMEM106a expression was suppressed in renal cancer cell lines

We started to profile the expression of TMEM106a by qPCR in both primary renal epithelial cells and renal cancer cells. Two normal renal cells were used as control: primary renal proximal tubule epithelial cell and primary renal cortical epithelial cell, which could represent the different structures of kidney. Five renal cancer cell lines were selected: 7860, 769-P, ACHN are renal adenocarcinoma cells, A-498 is renal carcinoma cell, and A-794 is adenocarcinoma cell. As shown in Fig. 1, TMEM106a mRNA level in primary renal proximal tubule epithelial cell was normalized as 1, the other normal renal epithelial cells showed similar level of TMEM106a. However, all the five renal cancer cell lines showed reduced level of TMEM106a to various extents. 786-0 and ACHN had the lowest levels of TMEM106a at about $20 \%$ of the level in control normal cells. The other three renal cancer cells showed about 50\% amount of mRNA compared to control cells. The results clearly indicated that TMEM106a mRNA was suppressed in renal cancer cells compared to normal renal cells. Since TMEM106a is a transmembrane protein, we also extracted the proteins from cell membrane and determined its presence level in different cell lines. As shown in Fig. 2, the membrane associated TMEM106a was significantly lower in cancer cells than in normal renal epithelial

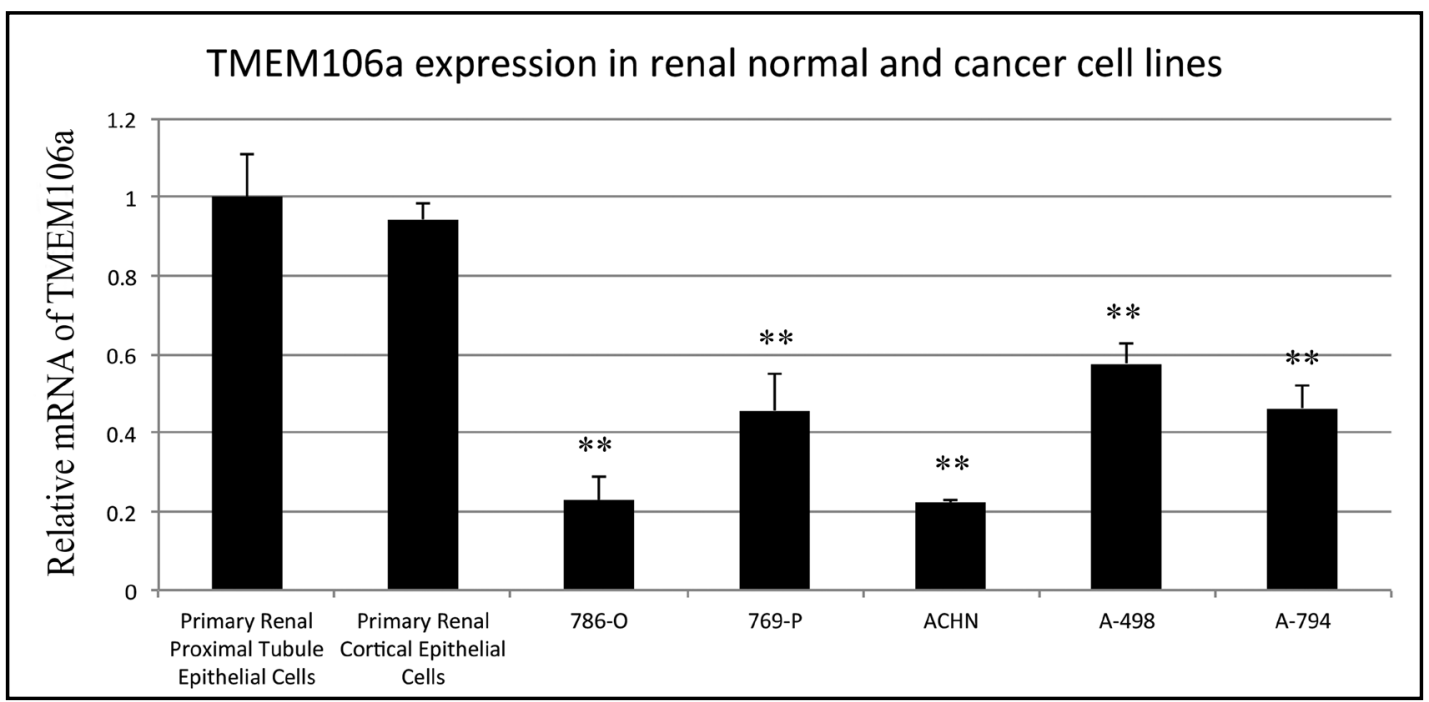

Fig. 1. TMEM106a mRNA expression level is suppressed in renal cancer cell lines. Two primary renal epithelial cells and five renal cancer cells (786-0, 769-0, ACHN, A-498 and A794) were grown and harvested for mRNA extraction. TMEM106a gene expression was determined by real-time PCR. The relative expression of TMEM106a from the indicated cell lines was shown relative to the value from renal proximal tubule epithelial cells. Data were expressed as mean \pm S.D. $\left(n=3\right.$ biological replicates): ${ }^{*} \mathrm{p}<0.05,{ }^{* *} \mathrm{p}<0.01$ for cancer lines versus primary renal epithelial cells in Student's t test. 


\section{Kidney Blood Pressure Research}

cells. This is consistent with what we have observed at mRNA level, implying TMEM106a is transcriptional suppressed in renal tumor cells.

\section{Over-expression}

of

TMEM106a in renal cancer cells attenuated cell growth

To understand the role of TMEM106a in renal cancer cells, we chose the cells with relatively low level of TMEM106a mRNA and restored the level by overexpression with lentivirus system. Then the growth of different cells was assessed by CCK-8 kit. ACHN and 786-

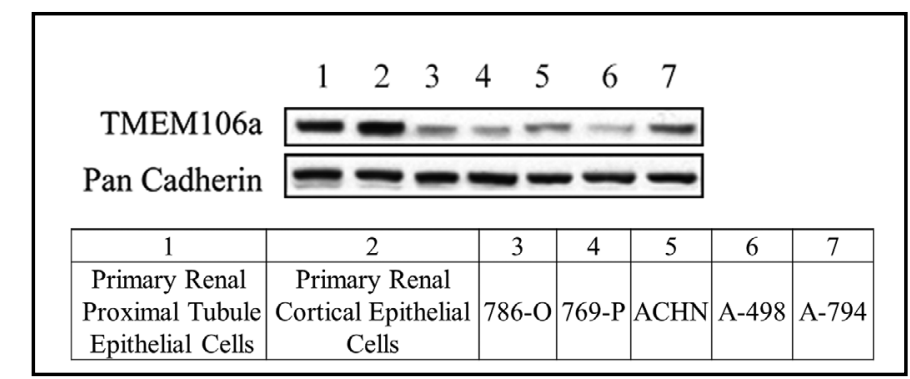

Fig. 2. TMEM106a protein level in cell membrane is lower in renal cancer cell lines. Two primary renal epithelial cells and five renal cancer cells (786-0, 769-0, ACHN, A-498 and A794) were grown and harvested for membrane protein extraction with Mem-PER Plus kit (Thermo Fisher Scientific). The membrane protein extract was resolved in SDS-PAGE and the presence of TMEM106a was determined by western blotting. The level of Cadherins (Pancadherin antibody \#4068 from Cell Signaling Technology) was used as loading control.

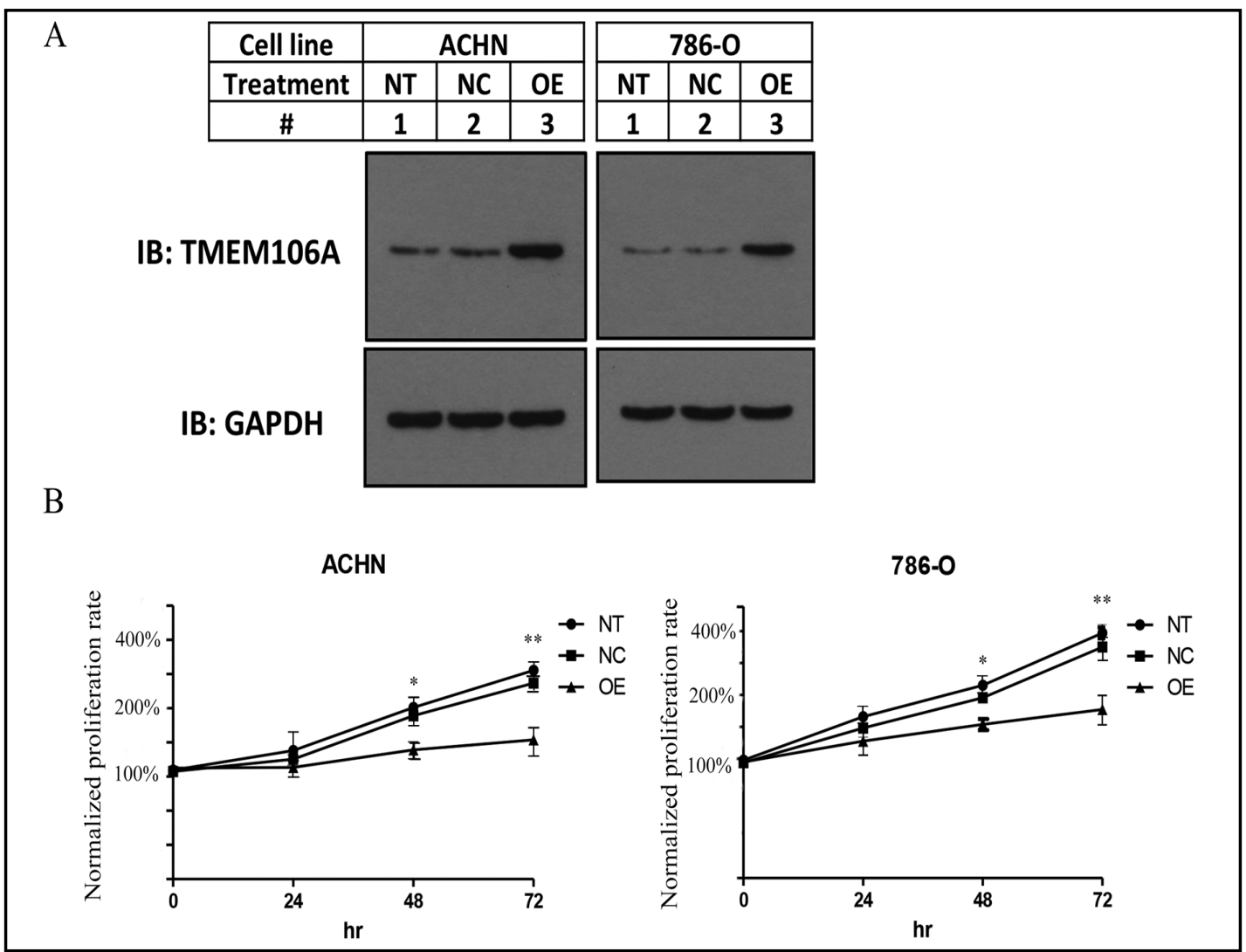

Fig. 3. The overexpression of TMEM106a in renal cancer cells reduces cell growth. (A) TMEM106a was over-expressed in ACHN and 786-0 cell lines with lentiviral expression system. Western blot analysis of TMEM106a from untransfected control (NT), empty vector transfected control (NC) and target gene transfected cells (OE) was shown. (B) After seeding the cells for $24 \mathrm{~h}$, the cell proliferation from the indicated lines was measured by CCK- 8 kit for indicated time points ( $24 \mathrm{~h}, 48 \mathrm{~h}$ and $72 \mathrm{~h}$ ). The data were presented as means \pm S.D. of at least three independent experiments: ${ }^{*} \mathrm{p}<0.05,{ }^{* *} \mathrm{p}<0.01$ for OE versus NT or NC. 


\section{Kidney Blood Pressure Research}

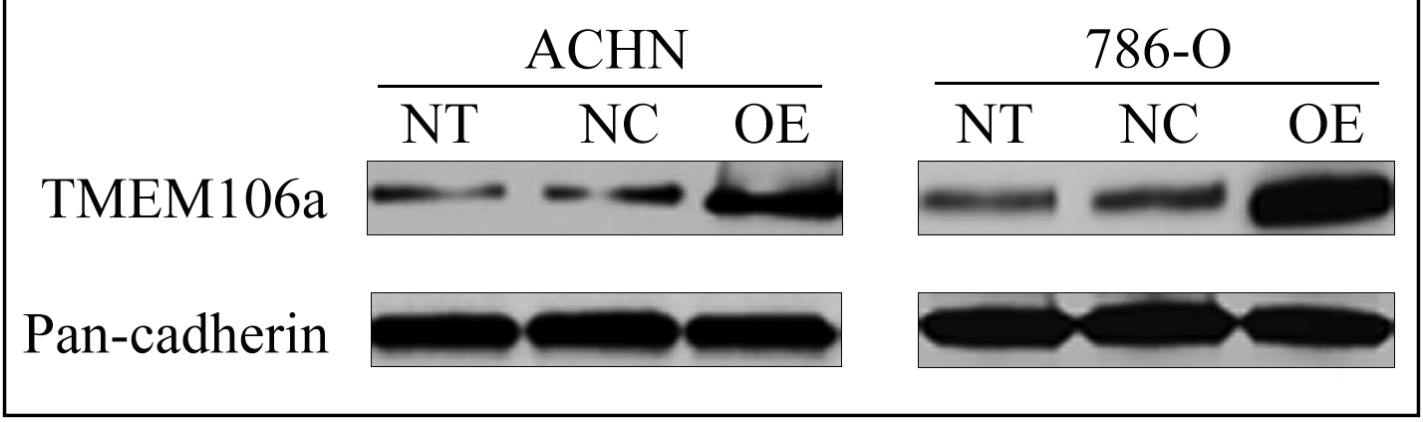

Fig. 4. Over-expressed TMEM106a is localized in cell membrane. TMEM106a was over-expressed in ACHN and 786-0 cell lines with lentiviral expression system. The membrane proteins were extracted with MemPER Plus kit. Western blot analysis of TMEM106a from untransfected control (NT), empty vector transfected control (NC) and target gene transfected cells (OE) was shown.

O cells with low TMEM106 were transduced with empty vector (NC) or overexpressing TMEM106a vector (OE) or left untransduced (NT), and the virus transduction was confirmed by Western blotting with anti-TMEM106a antibody. As shown in Fig. $3 \mathrm{~A}$, both cell lines with TMEM106a OE showed much stronger protein level than NT or NC which contained similar low level of TMEM106a proteins, suggesting the success of TMEM106a overexpression. In addition, we validated that overexpressed TMEM106a was localized in cell membrane as expected (Fig. 4). Then cell proliferation assay was performed for the different

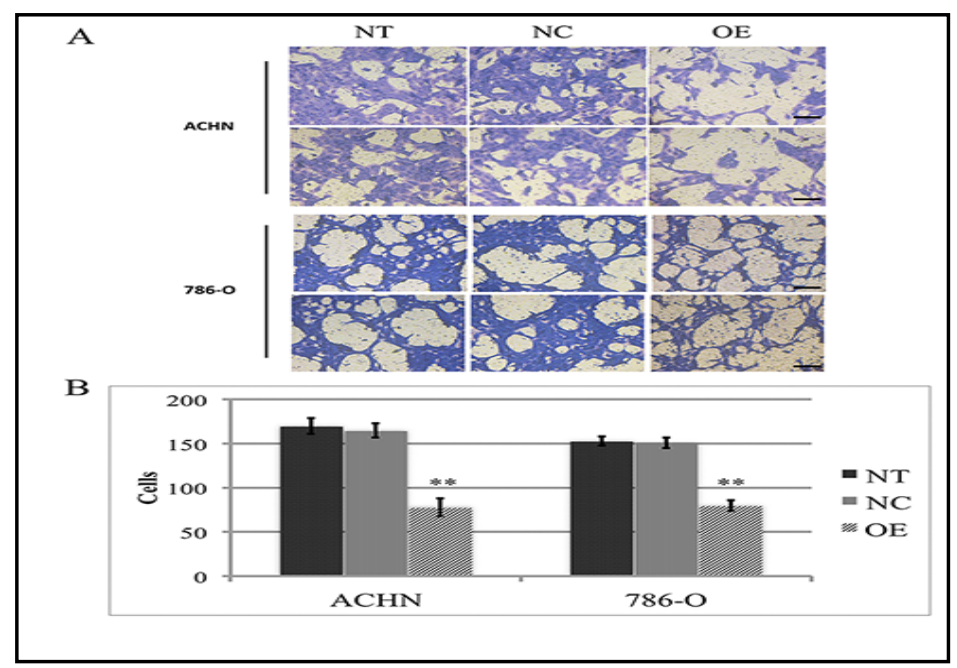

Fig. 5. The overexpression of TMEM106a inhibits human renal cancer cells migration. (A) Representative images of transwell migration study in ACHN or 786-O cell lines from each experimental group (NT, NC and $\mathrm{OE}$ ). The migrated cells in receiver plate were examined with Toluidine Blue 0 staining (scale bar: $40 \mu \mathrm{m}$ ). Experiments were repeated three times independently. (B) Quantitative results of total numbers of migrated cells from each experimental group of two cell lines. The data were presented as means \pm S.D. of three independent experiments: ${ }^{*} \mathrm{p}<0.05,{ }^{* *} \mathrm{p}<0.01$ for OE versus NT or NC. cell lines at the time points of $24 \mathrm{~h}, 48 \mathrm{~h}$, and $72 \mathrm{~h}$.

Consistently, both ACHN and 786-0 with TMEM106a overexpression showed slower growth rate compared to the NC and NT controls $(\mathrm{p}<0.01)$, as shown in Fig. 3B. The difference in growth rate was first observed at $48 \mathrm{~h}$ post measurement. The NC and NT controls showed similar trend of cell growth curve without much difference. This result demonstrated that overexpression of TMEM106a in the renal cancer cells could significantly attenuate the growth potential of cells.

Over-expression of TMEM106a inhibited the migration of human renal cancer cells

The challenge of renal cancer largely comes from the difficulty in early detection of the disease and many patients are diagnosed with renal cancer at quite late stages with metastasis to other organs. Therefore, we next investigated the function of TMEM106a in 


\section{Kidney Blood Pressure Research}

A
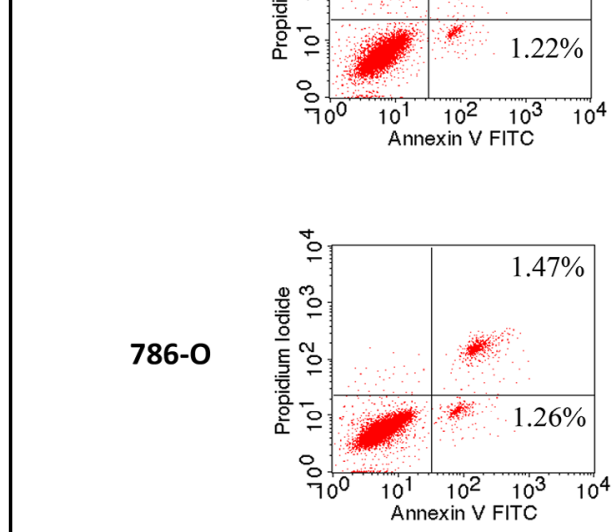

NT

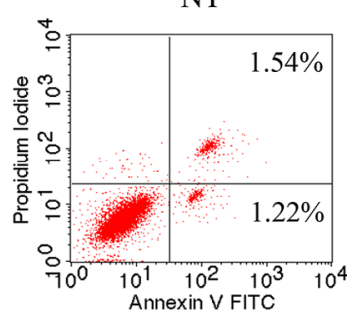

$.47 \%$

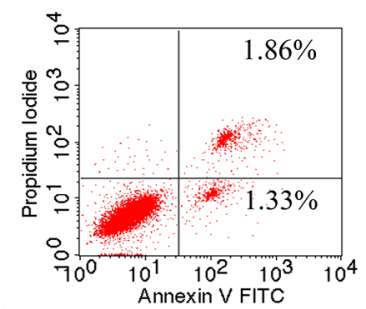

$\mathrm{NC}$
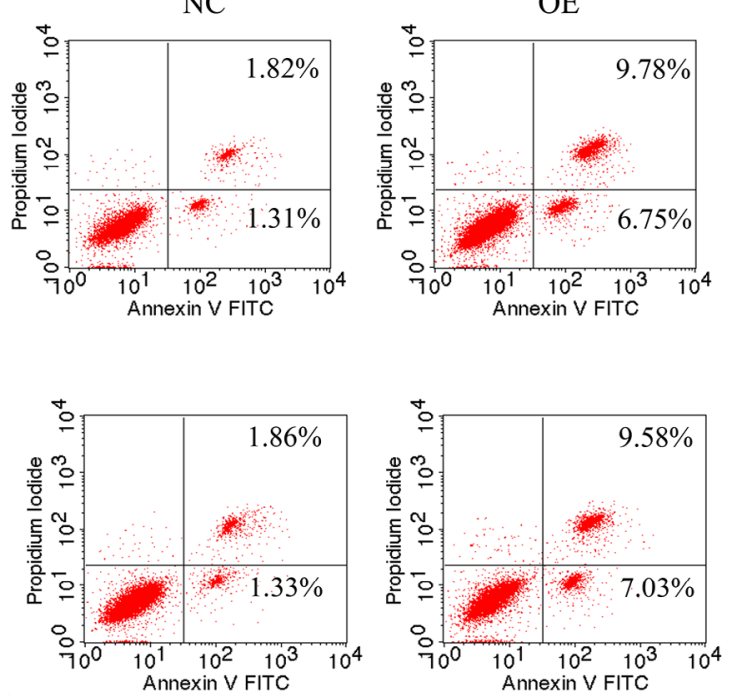

$\mathrm{B}$

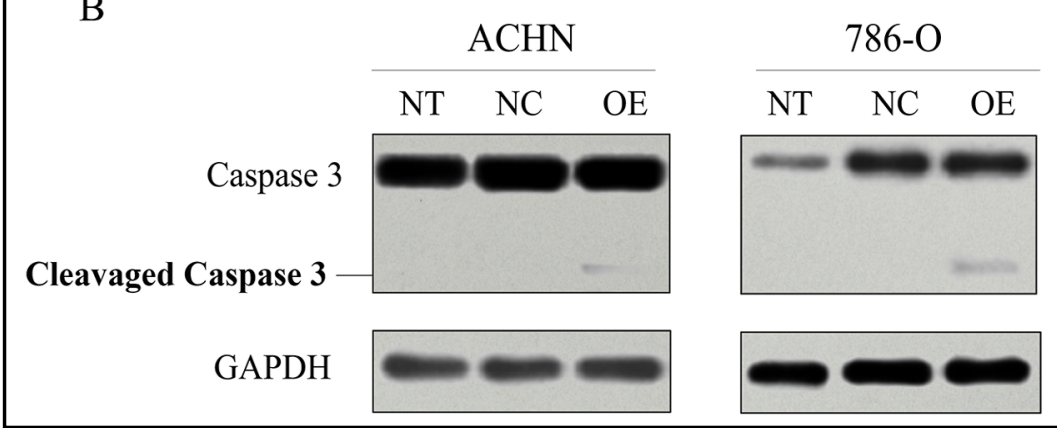

Fig. 6. The overexpression of TMEM106a in renal cancer cells activates caspase 3 dependent apoptosis pathway. (A) Representative result of flow cytometry analysis of ACHN or 786-0 lines from each experimental group (NC, NT and OE). Annexin V/PI staining was used to detect cell apoptosis. Experiments were repeated three times independently. (B) Caspase 3 activation in each experimental group was examined by western blot. Cleaved caspase-3 indicated the activation of caspase dependent apoptotic pathway. GAPDH was used as loading control. Experiments were repeated three times independently.

cell migration, which is essential for cancer metastasis. ACHN and 786-0 cells with different levels of TMEM106a were assessed in the trans-well assay, a well-established migration assay. As shown in Fig. 5A, the migrated cells in receiver plates were fixed and stained with Toluidine Blue 0 dye. TMEM106a OE ACHN and 786-O cells both showed the reduced number of cells after migration compared to NT or NC controls. The quantification of three independent experiments was shown in Fig. 5B, which clearly demonstrated the significant reduction of cell migration ability of the cells with TMEM106a overexpression compared to control cells, in both ACHN and 786-0 cell lines.

Over-expression of TMEM106a in renal cancer cells activated caspase 3 dependent apoptosis pathway

Cell apoptosis is a critical pathway for cell homeostasis and remains the number one target for cancer therapy, although cancer cells often find ways to evade the fate of apoptosis under drug treatment. We next investigated the function of TMEM106a in renal cancer apoptosis. Using the standard Annexin/Propidium Iodide staining method, ACHN and 786-0 cells with different levels of TMEM106a were subject to FACS analysis. As shown in Fig. 6A, 


\section{Kidney Blood Pressure Research}

A

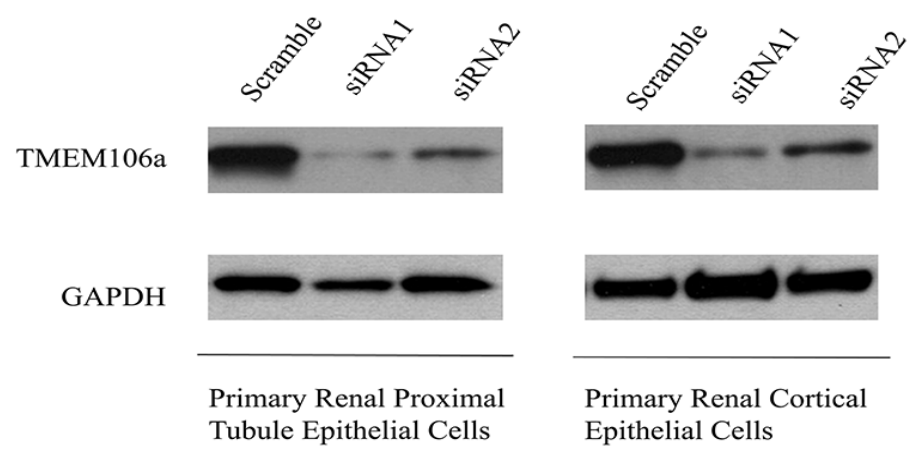

B

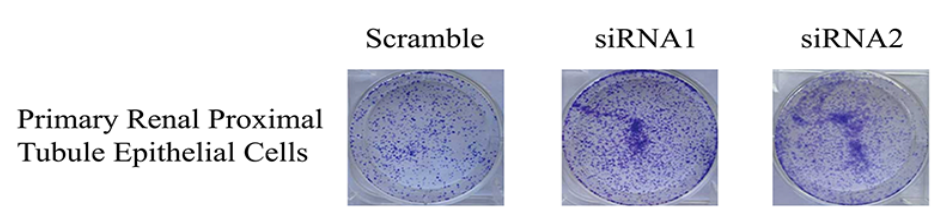

Primary Renal Cortical Epithelial Cells

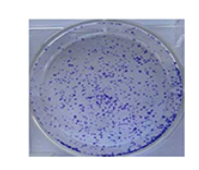

$\mathrm{C}$
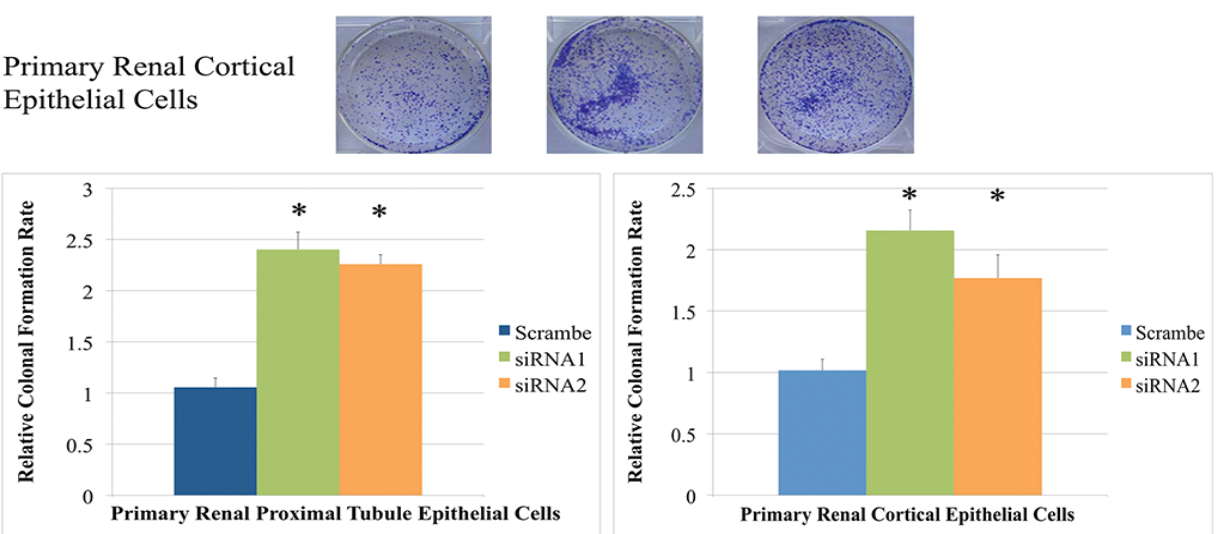

Fig. 7. Knocking-down TMEM106a in primary renal cells promotes clonal formation. (A) Western blot analysis of TMEM106a expression in primary renal epithelial cells transfected with scramble or either two siRNAs targeting human TMEM106a. GAPDH was used as loading control. (B) Representative image of clonal formation of primary renal epithelial cells transfected with scramble or either two siRNAs targeting human TMEM106a. Experiments were repeated three times independently. (C) Quantitative results of the relative clonal formation rate of cells transfected with TMEM106a siRNAs against control cells (scramble). The data were presented as means \pm S.D. of three independent experiments: ${ }^{*} \mathrm{p}<0.05,{ }^{* *} \mathrm{p}<0.01$ versus control.

NT or NC control cells showed only 1.22-1.33\% population with apoptosis, while TMEM106a OE cells showed $6.75 \%$ to $7.03 \%$ population with apoptosis respectively. Moreover, the necrotic cell population was also significantly elevated to about $10 \%$ in $\mathrm{OE}$ cells compared to the level of less than $2 \%$ in NC or NT controls. The FACS results were supported by the caspase 3 cleavage as shown in Fig. 6B. In both ACHN and 786-O cells with TMEM106a OE, the cleaved caspase 3 was readily detected, while barely seen in NT or NC control cells. These results suggested that TMEM106a promoted the cell apoptosis in renal cancer cell once overexpressed.

Down-regulation of TMEM106a in primary renal cells promoted colony formation ability.

To further reveal the function of endogenous TMEM106a protein in renal cells, we then knocked down the protein level of TMEM106a with siRNA in the primary renal epithelial cells with relatively high TMEM106a level. Firstly, the knockdown effect of TMEM106 by siRNA was confirmed by Western blotting method, as shown in Fig. 7A. Both siRNA 1 


\section{Kidney \\ Blood Pressure Research}

and siRNA2 could significantly decrease the level of endogenous TMEM106a proteins in two primary renal epithelial cell lines, compared to scrambled control siRNA. Next, we measured the cell growth potential by clonogenic assay as shown in Fig. 7B and 7C. TMEM106a knockdown cells showed enhanced ability to form colonies compared to scrambled controls in both primary renal epithelial cell lines. The quantification of Fig. 7B was shown in Fig. 7C which demonstrated the significance of difference $(p<0.01)$. In addition, we also measured the proliferation and migration in these TMEM106a knockdown cells. As shown in Fig. 8A, knocking down TMEM106a in both primary renal epithelial lines increased their proliferation capacity in comparison with controls. The difference was first observed at $48 \mathrm{~h}$ post measurement. In addition, knocking down TMEM106a also promoted cell migration in transwell assay (Fig. 8). These results further revealed TMEM106a as a tumor suppressor gene candidate. Therefore, knocking-down experiments confirmed the previous results with overexpressed TMEM106a cells that high level of TMEM106a led to attenuation of cell growth, migration and elevated apoptosis, while down-regulation of TMEM106a enhanced the cell growth potential. To elucidate the mechanistic basis of TMEM106a in tumorigenesis, we also sought to measure the impact of the change of TMEM106a expression on its downstream pathways in both renal cancer and normal cells [15]. In over-expression model, we found JNK phosphorylation was upregulated compared with control (Fig. 9A). In line with this result, knocking down TMEM106a in primary renal cells reduced JNK activation at basal level (Fig. 9B). Since JNK activation antagonises cell proliferation while its downregulation is implicated in tumorigenesis, this mechanistic study revealed that TMEM106a-JNK axis was implicated in human renal cell tumorigenesis.

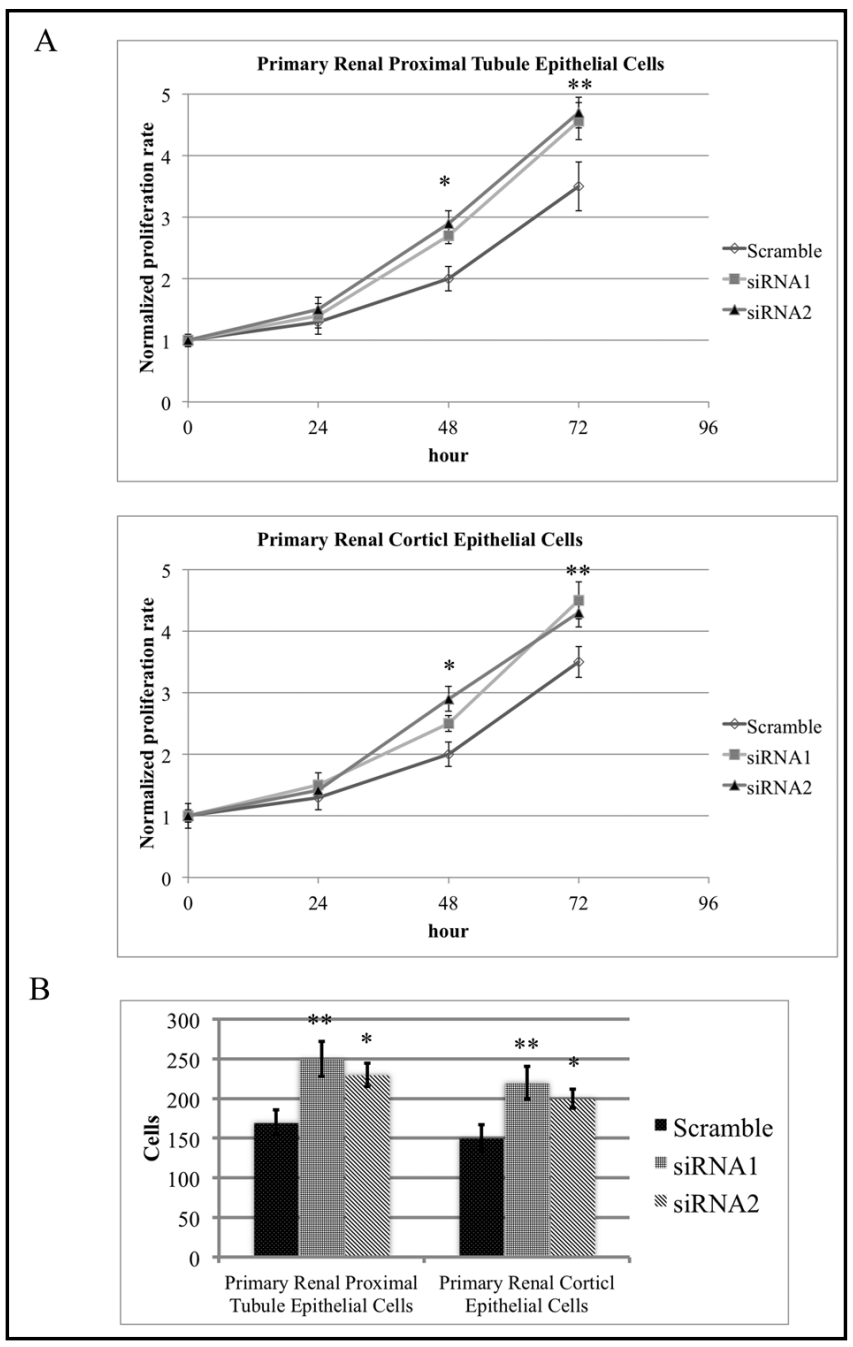

Fig. 8. Knocking-down TMEM106a in primary renal cells promotes cell proliferation and migration. (A) After seeding the cells for $24 \mathrm{~h}$, the cell proliferation for the indicated lines (transfected with siRNA1, siRNA2 against TMEM106a or scramble siRNA) was measured by CCK-8 kit at indicated time points ( $24 \mathrm{~h}, 48 \mathrm{~h}$ and $72 \mathrm{~h}$ ). The data were presented as means \pm S.D. of at least three independent experiments: ${ }^{*} \mathrm{p}<0.05$, ${ }^{* *} \mathrm{p}<0.01$ for TMEM106a siRNA transfected versus scramble. (B) Quantitative results of total numbers of migrated cells from each experimental group in transwell migration study. The data were presented as means \pm S.D. of three independent experiments: ${ }^{*} \mathrm{p}<0.05,{ }^{* *} \mathrm{p}<0.01$ for TMEM106a siRNA transfected versus scramble. 


\section{Kidney Blood Pressure Research}

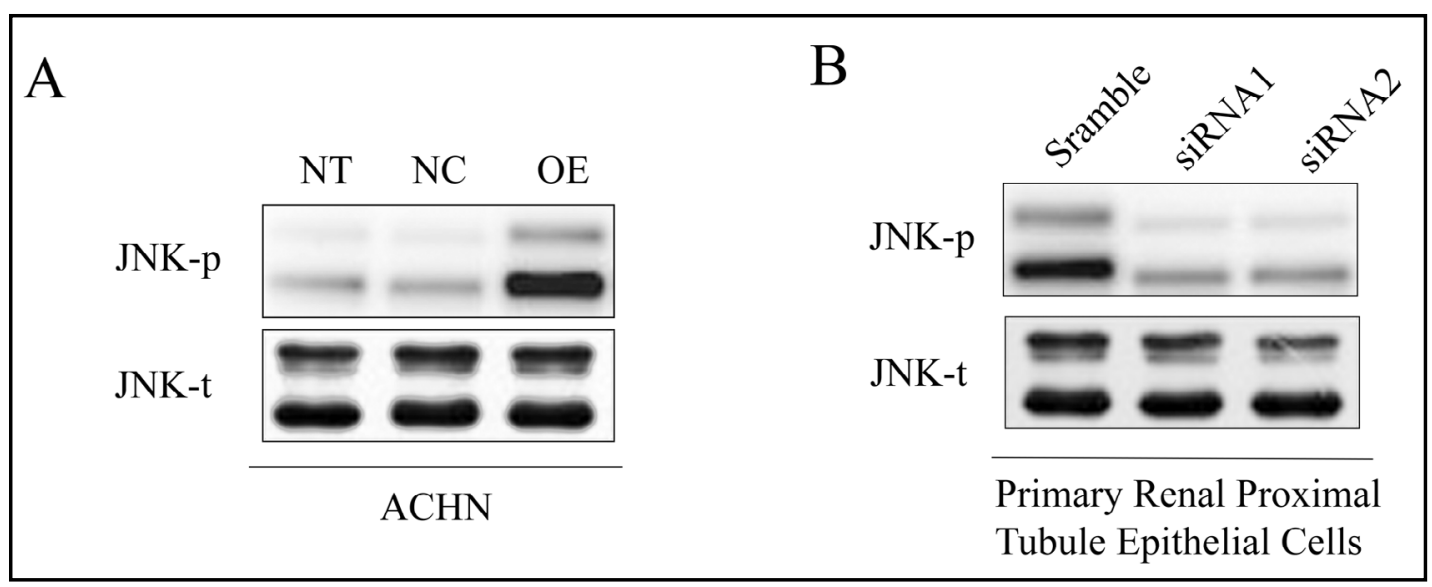

Fig. 9. The effect of over-expressing or knocking-down TMEM106a on JNK activation. (A) TMEM106a was over-expressed in ACHN line. The cell lysate was harvested and western blot analysis of JNK activation from untransfected control (NT), empty vector transfected control (NC) and target gene transfected cells (OE) was shown. (B) Western blot analysis of JNK activation in primary renal epithelial cells transfected with scramble or either two siRNAs targeting human TMEM106a.

\section{Discussion}

Renal cancer is a public health challenge worldwide as it is difficult to be detected in early stages. Recent progress has identified several key factors and pathways responsible for renal tumorigenesis such as VHL, VEGF, NFkB, mTOR pathways [7, 11, 22]. However, there is still a big gap to unveiling the detailed molecular mechanism of renal cancer and identifying potential target for therapeutic application. Here in this study we demonstrate that TMEM106a acts as a novel tumor suppressor in renal cancer, and it is frequently downregulated in renal cancer cell lines. Restoration of TMEM106 expression in renal cancer cells led to attenuated cell proliferation and migration, as well as enhanced cell apoptosis compared to control cells. On the contrary, TMEM106a knockdown by siRNA in primary renal cells showed increased colony formation compared to the control cells with scrambled siRNA transfection, which supported the results obtained by overexpression study.

TMEM106a is a type II transmembrane protein with relatively unclear function in human. Our study for the first time demonstrated that TMEM106a was an important tumor suppressor in renal cancer. This conclusion was consistent with the finding in gastric cancer that TMEM106a was suppressed by promoter methylation and restoration of TMEM106a attenuated the tumor growth in xenograft model [14]. As TMEM106 is also expressed in kidney tissues, we firstly examined the expression profile in multiple renal cancer cell lines compared with primary renal cells and the result revealed that TMEM106a was downregulated in tumor cells in contrast to normal cells. How TMEM106a was repressed in renal cancer is still unclear. We can speculate that promoter methylation might be one of the reasons, similar to the situation in gastric cancer. It is of great importance to understand the detailed mechanism of TMEM106a loss in cancers as it may uncover the potential method to prevent or revert the deleterious change. This will be further investigated in the future.

TMEM106a overexpression in renal cancer cells caused dramatic consequences in cell proliferation and migration as shown in the results of this study. Similar results from previous gastric cancer research lend support to our findings [14]. However, the mechanism responsible for the phenotypic change was largely elusive. As TMEM106a is a highly conserved transmembrane protein in mammals, we may obtain some hint from mouse TMEM106a study. Although the ligand to bind TMEM106a is still unknown, Chen 


\section{Kidney Blood Pressure Research}

and colleagues successfully generated antibody against TMEM106a which could bind and activate TMEM106a dependent downstream signaling pathways in macrophages, including MAPK, NFKB and JAK-STAT pathways [15]. All these pathways play essential roles to regulate a plethora of cellular processes such as cell growth and migration, as well as cell apoptosis [23-25]. The activation patterns were at large similar to those elicited by lipopolysaccharide (LPS) stimulation, suggesting that they may share some common modules of signaling transduction from membrane receptor engagement. However, it is unclear whether TMEM106a exerts its function in cancer cells through the similar activation mechanism. The detailed mechanism will be the focus of future study.

Since manipulation of TMEM106a expression level in cancer cells could result in the significant biological consequences, we tend to believe that TMEM106a may undergo autoactivation once overexpressed in cancer cells, and switch on the downstream pathways. Also it is possible that some endogenous TMEM106a ligands remain to be identified on the surface of neighbouring cells or in the cancer microenvironment. In addition to its role in cancer cells, TMEM106a may regulate various cell types as suggested in mouse macrophages. As we know, tumor associated macrophages actively promote all aspects of tumor initiation, growth and metastasis. Given the function of TMEM106a in macrophage development and polarization, it will be very intriguing to study the interaction of tumor cells and infiltrated immune cells in a more physiological dimension. To address this question, it is necessary to establish the mouse models with genetic manipulation of TMEM106a in different compartments involved in tumorigenesis, such as epithelial cells, immune cells, endothelial cells.

\section{Conclusion}

In summary, TMEM106a plays an important role in renal cancer pathogenesis as a potential tumor suppressor. Its inactivation contributes to the enhanced tumor growth and migration while at the same time attenuating cell apoptosis possibly via multiple signaling pathways such as MAPK, NFKB and JAK-STAT pathways.

\section{Disclosure Statement}

None of the authors declare competing financial interests.

\section{Acknowledgements}

This project was supported by the Shenzhen Science and Technology Project (grant nos. JSGG 20160301162913683 and JCYJ 20140414170821273 ) and Fund for High Level University Construction of Medical Discipline (2016031638), China.

\section{References}

1 Hemmelgarn BR, Manns BJ, Lloyd A, James MT, Klarenbach S, Quinn RR, Wiebe N, Tonelli M, Alberta Kidney Disease Network: Relation between kidney function, proteinuria, and adverse outcomes. JAMA 2010;303:423-429.

2 Jemal A, Siegel R, Xu J, Ward E: Cancer statistics, 2010. CA Cancer J Clin 2010;60:277-300.

-3 MacLennan S, Imamura M, Lapitan MC, Omar MI, Lam TB, Hilvano-Cabungcal AM, Royle P, Stewart F, MacLennan G, MacLennan SJ, Canfield SE, McClinton S, Griffiths TR, Ljungberg B, N’Dow J, Group USRR, Panel EAURCG: Systematic review of oncological outcomes following surgical management of localised renal cancer. Eur Urol 2012;61:972-993. 


\section{Kidney \\ Blood Pressure Research}

Wu et al.: Role of TMEM106a in Renal Cancer

4 Jonasch E, Gao J, Rathmell WK: Renal cell carcinoma. BMJ 2014;349:g4797.

5 Capitanio U, Montorsi F: Renal cancer. Lancet 2016;387:894-906.

6 Rini BI, Atkins MB: Resistance to targeted therapy in renal-cell carcinoma. Lancet Oncol 2009;10:992-1000.

7 Rini BI, Campbell SC, Escudier B: Renal cell carcinoma. Lancet 2009;373:1119-1132.

-8 Calzada MJ, Esteban MA, Feijoo-Cuaresma M, Castellanos MC, Naranjo-Suarez S, Temes E, Mendez F, YanezMo M, Ohh M, Landazuri MO: Von hippel-lindau tumor suppressor protein regulates the assembly of intercellular junctions in renal cancer cells through hypoxia-inducible factor-independent mechanisms. Cancer Res 2006;66:1553-1560.

-9 Kaelin WG, Jr: The von hippel-lindau tumour suppressor protein: 02 sensing and cancer. Nat Rev Cancer 2008;8:865-873.

10 Yang L, Zhao Z, Zhao S, Chen C, Cong X, Li Z, Ren M: The clinicopathological significance of epigenetic silencing of vhl promoter and renal cell carcinoma: A meta-analysis. Cell Physiol Biochem 2016;40:14651472.

11 Koul H, Huh JS, Rove KO, Crompton L, Koul S, Meacham RB, Kim FJ: Molecular aspects of renal cell carcinoma: A review. Am J Cancer Res 2011;1:240-254.

12 Liu X, Wang J, Sun G: Identification of key genes and pathways in renal cell carcinoma through expression profiling data. Kidney Blood Press Res 2015;40:288-297.

13 Tian X, Dai S, Sun J, Jiang S, Sui C, Meng F, Li Y, Fu L, Jiang T, Wang Y, Su J, Jiang Y: Inhibition of mdm2 re-sensitizes rapamycin resistant renal cancer cells via the activation of $\mathrm{p} 53$. Cell Physiol Biochem 2016;39:2088-2098.

14 Xu D, Qu L, Hu J, Li G, Lv P, Ma D, Guo M, Chen Y: Transmembrane protein 106a is silenced by promoter region hypermethylation and suppresses gastric cancer growth by inducing apoptosis. J Cell Mol Med 2014;18:1655-1666.

15 Dai H, Xu D, Su J, Jang J, Chen Y: Transmembrane protein 106a activates mouse peritoneal macrophages via the mapk and nf-kappab signaling pathways. Sci Rep 2015;5:12461.

-16 Lang CM, Fellerer K, Schwenk BM, Kuhn PH, Kremmer E, Edbauer D, Capell A, Haass C: Membrane orientation and subcellular localization of transmembrane protein 106B (TMEM106B), a major risk factor for frontotemporal lobar degeneration. J Biol Chem 2012;287:19355-19365.

17 Brady OA, Zheng Y, Murphy K, Huang M, Hu F: The frontotemporal lobar degeneration risk factor, tmem106b, regulates lysosomal morphology and function. Hum Mol Genet 2013;22:685-695.

18 Vass R, Ashbridge E, Geser F, Hu WT, Grossman M, Clay-Falcone D, Elman L, McCluskey L, Lee VM, Van Deerlin VM, Trojanowski JQ, Chen-Plotkin AS: Risk genotypes at tmem106b are associated with cognitive impairment in amyotrophic lateral sclerosis. Acta Neuropathol 2011;121:373-380.

19 Rutherford NJ, Carrasquillo MM, Li M, Bisceglio G, Menke J, Josephs KA, Parisi JE, Petersen RC, Graff-Radford NR, Younkin SG, Dickson DW, Rademakers R: Tmem106b risk variant is implicated in the pathologic presentation of alzheimer disease. Neurology 2012;79:717-718.

20 Assassi S, Reveille JD, Arnett FC, Weisman MH, Ward MM, Agarwal SK, Gourh P, Bhula J, Sharif R, Sampat K, Mayes MD, Tan FK: Whole-blood gene expression profiling in ankylosing spondylitis shows upregulation of toll-like receptor 4 and 5. J Rheumatol 2011;38:87-98.

-21 Genini S, Nguyen TT, Malek M, Talbot R, Gebert S, Rohrer G, Nonneman D, Stranzinger G, Vogeli P: Radiation hybrid mapping of 18 positional and physiological candidate genes for arthrogryposis multiplex congenita on porcine chromosome 5. Anim Genet 2006;37:239-244.

22 Rini BI: Vascular endothelial growth factor-targeted therapy in metastatic renal cell carcinoma. Cancer 2009;115:2306-2312.

23 Levy DE, Darnell JE, Jr: Stats: Transcriptional control and biological impact. Nat Rev Mol Cell Biol 2002;3:651-662.

24 Dhillon AS, Hagan S, Rath 0, Kolch W: Map kinase signalling pathways in cancer. Oncogene 2007;26:32793290.

25 Hoesel B, Schmid JA: The complexity of nf-kappab signaling in inflammation and cancer. Mol Cancer 2013;12:86. 